\title{
Comparative effects of fluid resuscitation on lung and heart oxidative injury in a rat model of hemorrhagic shock
}

\author{
Kubra VARDAR, Ugur AKSU
}

Istanbul University, Science Faculty, Department of Biology, Istanbul, Turkey

\section{ISTANBUL}

\section{ÜNIVERSITESI}

Background: Although fluid resuscitation therapy is the first step of treatment for hemorrhagic shock, there is still debate on the different outcome for each organ.

Objectives: In the present study, we aimed to compare the oxidative effects of unbalanced resuscitation fluids for lungs and heart in a rat hemorrhagic shock model.

Material and methods: In 24 anaesthetized, spontaneously ventilated rats, hemorrhagic shock was induced by withdrawing $\sim 50 \%$ of total blood volume from the femoral artery until mean arterial pressure (MAP) was reduced to $40 \mathrm{mmHg}$. One hour after reaching a MAP of $40 \mathrm{mmHg}$, animals were resuscitated with either:

$$
\begin{aligned}
& \Rightarrow>\text { Colloid solution as Hydroxy ethyl starch }(6 \%)(n=6) \\
& \Rightarrow>\text { Crystalloid solution as } 0.9 \% \mathrm{NaCl}(n=6)
\end{aligned}
$$

until a target MAP of $80 \mathrm{mmHg}$ was reached throughout one hour. Hemolysis index, hematocrit, and catalytic iron were measured in plasma and major lipid peroxidation product as malondialdehyde (MDA) levels in both lungs and heart were measured by spectrophotometric determination following experimental procedure.

Results: No significant changes in withdrawn blood volume were found in resuscitation groups. However, the amount of fluid to achieve the target blood pressure were heigher in crystalloid group. Furhermore, hemotocrit levels in crystalloid group were similar in colloid group at the end of experiment. Hemorrhagic shock increased MDA levels in lung tissues but not in heart tissues. While both colloid and crystalloid resuscitation suppressed MDA levels in lung tissues, lipid peroxidation was further increased by crystalloid resuscitation in heart tissues. Hemolysis index and plasma catalytic iron levels were increased in crystalloid group.
Conclusions: Lungs seem to be early effected organ than heart during hemorrhagic shock, and crystalloid resuscitation could start a stress via catalytic iron that is not already present. Hence organ based fluid resuscitation could be taken into consideration.

Table 1: Mean arterial pressure in groups

\begin{tabular}{|l|c|c|c|}
\hline & Baseline & Shock & Resuscitation \\
\hline MAP (mmHg) & & & \\
\hline Control & $103.5 \pm 7.8$ & $97.7 \pm 8.7$ & $101 \pm 7.9$ \\
\hline Shock & $109.4 \pm 6.0$ & $39.5 \pm 1.5$ & $31.8 \pm 0.7$ \\
\hline Shock + Colloid & $99.0 \pm 7.1$ & $45.0 \pm 2.6$ & $83.2 \pm 1.2$ \\
\hline Shock + Crystalloid & $117.5 \pm 5.2$ & $42.1 \pm 2.3$ & $78.2 \pm 3.1$ \\
\hline
\end{tabular}
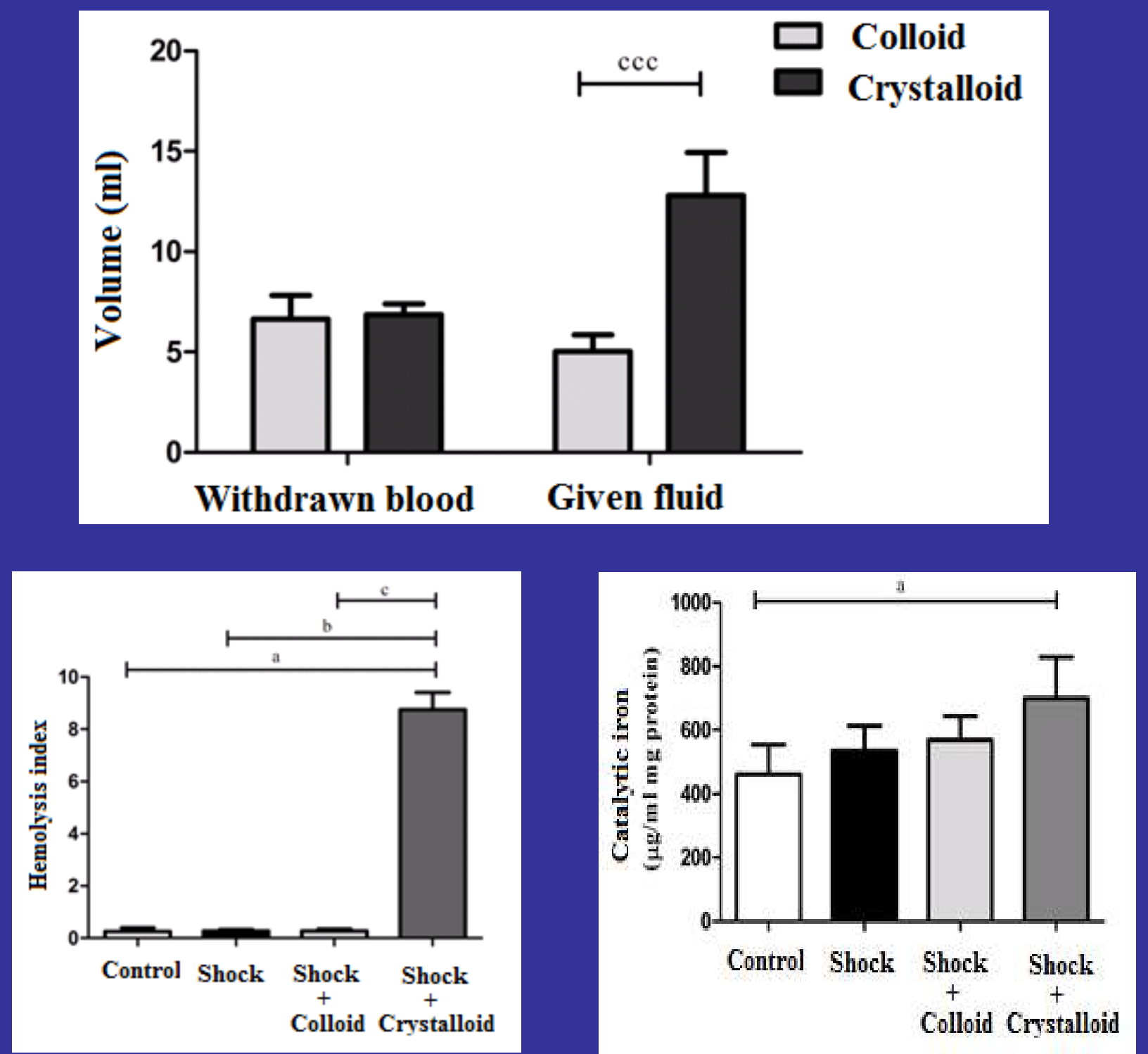
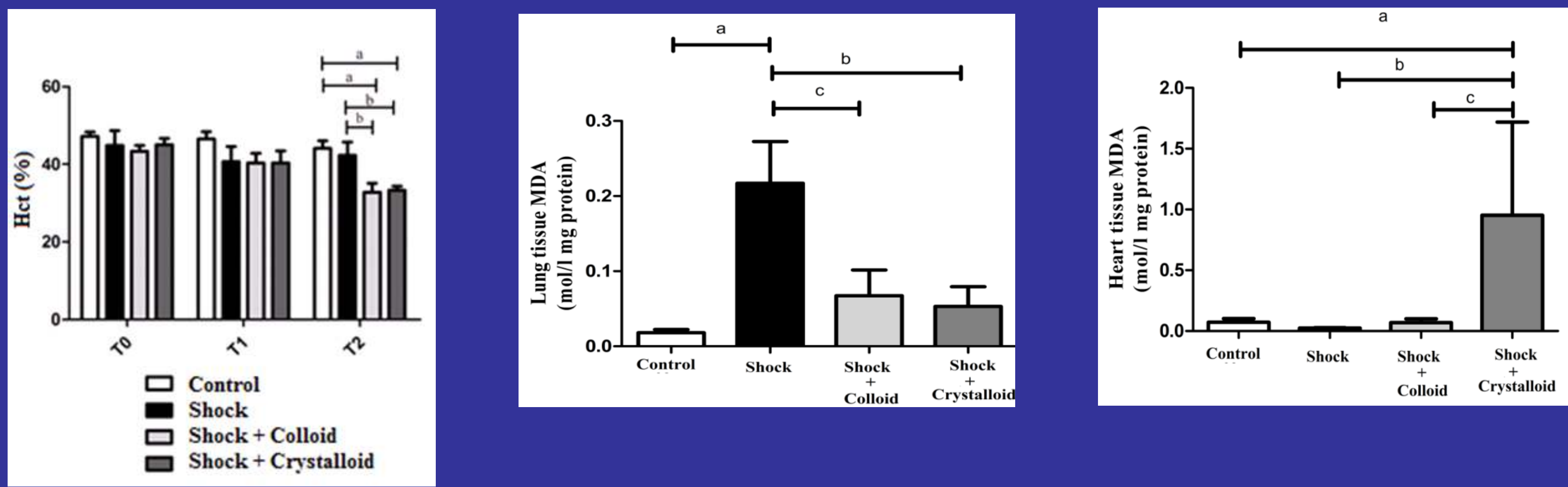